\title{
USO DO PLANEJAMENTO DE CENÁRIOS NO FUZZY FRONT END: APLICAÇÃO DA METODOLOGIA GBN EM UMA EMPRESA DO SETOR AUTOMOTIVO
}

\author{
Caroline Gava (gavacaroline@ gmail.com) - Universidade Federal de Minas Gerais \\ Isabela Vanice Pereira Oliveira (isabelavpo@gmail.com) - Universidade Federal de Minas Gerais \\ Júlia Araújo Tiso Mudrik (juliatiso@gmail.com) - Universidade Federal de Minas Gerais \\ Ana Paula Pena Almeida (anapaula.appa@gmail.com) - Universidade Federal de Minas Gerais \\ Raoni Barros Bagno (rbagno@ dep.ufmg.br) - Universidade Federal de Minas Gerais
}

\section{RESUMO}

O Fuzzy Front End (FFE), ou pré-desenvolvimento, é uma etapa crítica para o desenvolvimento bemsucedido de novos produtos. Apesar dos progressos realizados em estudos teóricos e empíricos, os desafios para gerir o FFE ainda são inúmeros, principalmente devido aos elevados graus de incerteza e complexidade da fase. Nesse contexto, várias técnicas e ferramentas foram propostas para dar suporte à etapa, entre elas, o Planejamento de Cenários (PC). O objetivo deste trabalho é explorar as potenciais contribuições do PC para os desafios do FFE identificados pela literatura. A partir de um estudo de caso realizado em uma grande indústria automotiva, em que a metodologia de PC desenvolvida pela Global Business Network (GBN) foi utilizada como metodologia central do processo de planejamento estratégico de novos produtos, identificou-se a promoção da integração entre as diferentes áreas funcionais, o aumento do número e da robustez das ideias discutidas, e a descentralização da identificação de oportunidades como os principais benefícios trazidos pelo uso do PC. O estudo apresenta em detalhes o processo de aplicação da metodologia no contexto da empresa estudada e realiza recomendações para potencializar as suas contribuições em aplicações futuras, em especial em relação aos desafios do FFE que não foram totalmente suportados no caso em questão. Além da observação direta, foram realizados, ao final do estudo, entrevistas e questionários com os colaboradores que participaram de pelo menos $85 \%$ dos workshops da metodologia da GBN. Esse levantamento permitiu avaliar a percepção dos participantes sobre as contribuições trazidas pelo PC, robustecendo as análises e os resultados previamente encontrados.

Palavras-chave: Processo de Desenvolvimento de Produtos; Fuzzy Front End; Pré-desenvolvimento; Planejamento de Cenários; Global Business Network, Indústria Automotiva.

Área: Ferramentas e métodos de desenvolvimento de produtos e serviços

\section{INTRODUÇÃO}

A gestão da inovação e do Processo de Desenvolvimento de Produtos (PDP) ocupa uma posição central na academia e na prática das empresas. A globalização da economia, o aumento da complexidade tecnológica, a redução do ciclo de vida dos produtos, entre vários outros fatores, exige que as empresas inovem de forma continua e eficaz para que se mantenham competitivas e sobrevivam no mercado (TIDD et al., 2008).

O pré-desenvolvimento, ou Fuzzy Front End (FFE), é apresentado como a primeira fase do PDP e inclui a formulação e comunicação das estratégias de produto, a identificação e avaliação de oportunidades, a geração de ideias e a definição do conceito dos produtos (KHURANA; ROSENTHAL, 1998). A fase é especialmente importante para o 
desenvolvimento de produtos inovadores, tipicamente associados a elevados níveis de incerteza e complexidade (HOLAHAN et al., 2014), e para os quais os modelos gerenciais tradicionais tendem a não fornecer o suporte necessário (KATZ, 2011). Nesses casos, esforços anteriores orientados para a redução das incertezas podem ser realizados antes de iniciar o processo de desenvolvimento formal (i.e. o PDP) (MOENAERT et al., 1995).

Apesar dos progressos realizados em estudos teóricos e empíricos, os desafios para gerir o FFE ainda são inúmeros. Alguns deles estão relacionados ao gerenciamento das ideias (e.g. orientação apenas para o curto prazo, centralidade, incerteza quanto aos resultados, falta de ideias adequadas) e ao gerenciamento da atenção, em especial diante das limitações cognitivas dos tomadores de decisão (POSTMA et al., 2012).

Diante deste contexto, várias técnicas e ferramentas foram propostas por acadêmicos e profissionais para dar suporte às atividades e aos desafios do FFE, entre elas o Planejamento de Cenários (PC) (JETTER, 2003; COSTA; TOLEDO, 2016). O PC é um método centrado no processo de criação e exploração de diferentes futuros projetados, de forma a identificar riscos e oportunidades em questões estratégicas para as organizações (SCHOEMAKER, 1995). Vários autores propõem abordagens distintas para o processo de PC, desde a apresentação do conceito até à construção dos cenários em si. Uma das metodologias de destaque é a da Global Business Network (GBN), consultoria americana entre as líderes de mercado.

Postma et al. (2012) exploraram em profundidade as contribuições do PC para aumentar a qualidade e a efetividade do FFE, apresentando as funções do PC como possíveis soluções para os problemas identificados na fase. Os autores concluíram, entre outros, que o PC pode realçar a criatividade no FFE ao apresentar possíveis e relevantes acontecimentos externos futuros e ao se concentrar apenas em incertezas críticas. Eles também destacam a necessidade de mais pesquisas empíricas em diferentes contextos para testar os resultados encontrados.

O objetivo deste trabalho foi explorar as potenciais contribuições do PC para o FFE e avaliar os fatores facilitadores e dificultadores do processo de aplicação da metodologia de PC da GBN. Baseado no trabalho de Postma et al. (2012), a relação das funções do PC com os problemas do FFE foram verificadas através de um estudo de caso em uma grande indústria automotiva. Todo o processo de uso da metodologia, incluindo as adaptações e dificuldades encontradas, são descritos e discutidos em detalhe.

A indústria automotiva detém um lugar de destaque na vanguarda de inovações tecnológicas e gerenciais sendo, historicamente, um setor proeminente quanto à adoção de métodos formais para a gestão do PDP. A importância dessa atividade para a indústria é atestada pela participação dos novos produtos no faturamento global das empresas. No Brasil, produtos lançados nos últimos três anos representam uma média de $47 \%$ das vendas anuais (MIGUEL, 2006).

Especialmente nas últimas décadas, a indústria automotiva tem experimentado um crescente aumento da complexidade e competitividade do mercado global, marcado por uma enorme segmentação de clientes, em termos de gostos e poder de compra, e por diferentes legislações locais que dificultam o uso de soluções globais (STURGEON et al., 2008). Esse contexto impulsiona as empresas a procurarem novas formas para aperfeiçoarem suas operações e diferenciarem seus produtos de forma eficiente e a custos competitivos (BACKMAN et al., 2007), trazendo impactos consideráveis ao seu PDP convencional e aumentando a importância dos esforços no pré-desenvolvimento.

\section{REFERENCIAL TEÓRICO}




\subsection{Fuzzy Front End (FFE)}

O FFE, muitas vezes chamado de pré-desenvolvimento do PDP, inclui a formulação e comunicação da estratégia do produto, a identificação e avaliação de oportunidades, a geração de ideias, a conceituação do produto e o planejamento de projetos (KHURANA; ROSENTHAL, 1998) (figura 1). Para Murphy e Kumar (1997), a fase consiste na geração de ideias, definição do produto e avaliação do projeto. Kim e Wilemon (2002) destacam a importância da seleção bem-sucedida das oportunidades e defendem que a missão da FFE é definir claramente o conceito de produto, uma vez que as atividades na fase de desenvolvimento (PDP) dependem diretamente dessas definições.

Figura 1. Modelo do FFE de Khurana e Rosenthal. Fonte: Traduzida de (KHURANA; ROSENTHAL, 1998)

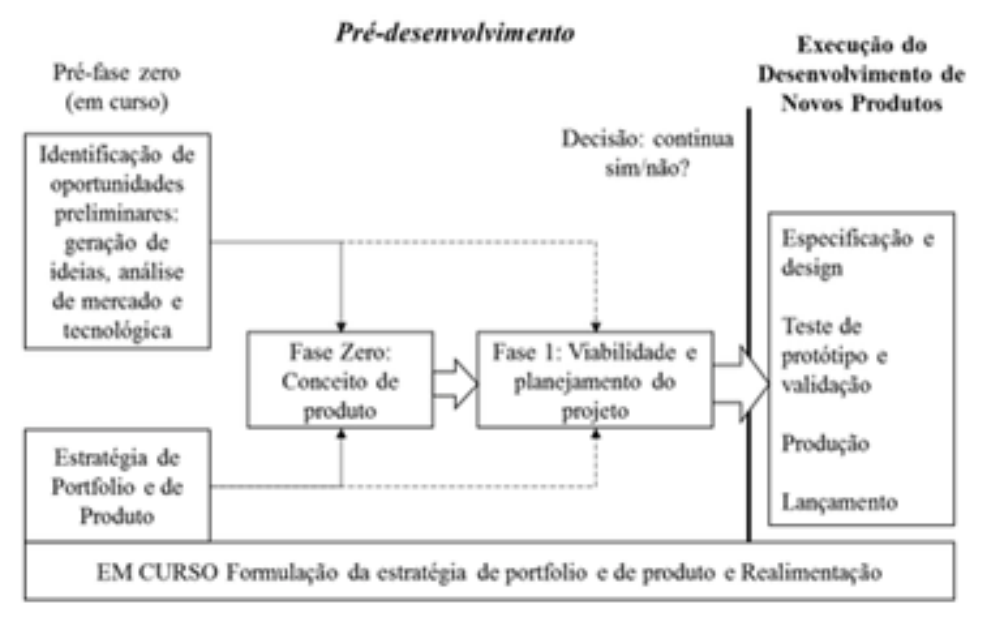

O FFE é reconhecidamente uma fase dinâmica e altamente incerta (KIM; WILEMON, 2002; JETTER, 2003) e, por isso, mais difícil de ser gerenciada. Baseados nos problemas relacionados ao gerenciamento de inovações identificados por Van de Ven (1986), Postma et al. (2012) colocam que dois deles destacam-se especialmente no FFE: o gerenciamento de ideias e gerenciamento de atenção. Apesar da complexidade associada a fase, vários autores defendem que o FFE representa uma grande oportunidade para redução do tempo e custo de desenvolvimento (KHURANA; ROSENTHAL, 1998) e contribui diretamente para o sucesso de um novo produto (COOPER, 1988; KOEN et al., 2001).

\subsection{Planejamento de Cenários (PC)}

O PC é um método disciplinado de imaginar possíveis futuros a partir da identificação de tendências e incertezas críticas sobre o ambiente de negócio de uma organização, oferecendo a ela um framework para um debate construtivo (SCHOEMAKER, 1995). Desenvolvido originalmente para o planejamento militar na II Guerra Mundial, o PC foi introduzido no campo industrial como uma ferramenta de planejamento estratégico pela Dutch/Shell Royal Group no início dos anos 1970 (VAN DER HEIJDEN, 1996).

Bood e Postma (1997) sintetizam cinco grandes funções do PC, nas quais as três primeiras se concentram no produto da análise de cenários (extraindo o valor dos cenários futuros resultantes), e as três últimas se concentram no processo de realização de análise de cenários (extraindo o valor do processo): (1) avaliação e seleção de estratégias; (2) integração de vários tipos de dados orientados para o futuro; (3) conscientização dos gerentes das incertezas do ambiente; (4) ampliação dos modelos mentais dos gestores; e (5) acionamento e aceleração dos processos de aprendizado organizacional. 
Existem várias metodologias de PC difundidas (BISHOP et al., 2007). Entre elas, estão: a metodologia implementada por Wack (1985a, 1985b) na Royal Dutch Shell; os dez passos para a construção de cenários de Schoemaker (1995); e também a abordagem da Global Business Network (GBN) (OGILVY; SCHWARTZ, 2004).

Ogilvy e Schwartz (2004), dentro da metodologia GBN, descrevem os cenários como narrativas de ambientes futuros alternativos, cujas decisões tomadas com base nessas percepções podem vir a ocorrer. Eles não são previsões, mas sim uma ferramenta de hipóteses de diferentes futuros projetados especificamente para destacar os riscos e oportunidades envolvidos em questões estratégicas específicas. Os autores apresentam a metodologia GBN em sete etapas, e defendem que no processo de construção de cenários é essencial a participação de uma equipe diversa, de diferentes níveis organizacionais e departamentos.

a) Identificação da questão central: estabelecimento do foco de interesse de discussão da empresa;

b) Brainstorming dos fatores chaves: identificação de forças de mudança e tendências chave a influenciar a organização, sociedade, tanto regional quanto globalmente;

c) Identificação das Forças Motrizes e de Incertezas Críticas: forças motrizes podem ser fenômenos de mudança lenta, situações restritas, tendências que já estão ocorrendo, ou movimentos aparentemente inevitáveis ou irreversíveis. Incertezas críticas são forças para as quais, ao contrário, não há uma visão clara de desdobramento no futuro;

d) Seleção da lógica de construção dos cenários: cenários podem ser construídos por uma combinação orgânica de forças motrizes e incertezas críticas, ou por uma abordagem dedutiva de priorização e cruzamento de todos os fatores garantindo cenários qualitativamente diferentes de um modo lógico e não aleatório;

e) Concretização dos Cenários: construção da narrativa que dará robustez aos cenários, podendo ser utilizadas ferramentas como storytelling e personagens;

f) Determinação das implicações de cada cenário: o output desta etapa é conjunto de ações estratégicas decorrentes da avaliação dos cenários, que pode ser realizada pelas explorações das diferentes lógicas dos cenários;

g) Wild Cards: etapa de monitoramento e acompanhamento dos cenários a partir de acontecimentos chaves.

\subsection{Contribuição de planejamento de cenários para o FFE}

Na literatura, o uso do PC no FFE é indicado, principalmente, nas atividades de identificação de oportunidades e geração e enriquecimento de ideias (POSTMA et al., 2012; COSTA; TOLEDO, 2016). (JETTER, 2003) defende o uso do PC para o gerenciamento do FFE uma vez que os cenários endereçam o problema da incerteza sobre os desenvolvimentos futuros de forma holística, ou seja, ao invés de tentar prever o estado futuro de algum elemento crítico, eles consideram um número possível de ambientes futuros.

Postma et al. (2012) realizaram uma exploração aprofundada das contribuições do PC para o FFE ao identificar um extenso conjunto de problemas do FFE para os quais a análise de cenários pode ser útil. Eles relacionam as principais funções do PC com os problemas do FFE para demonstrar como a análise de cenários pode melhorar a capacidade de gerenciamento da fase (figura 1). A setas na figura 2 indicam quais funções contribuem para a solução de cada um dos problemas do FFE. 
Figura 2. Contribuição do PC para o FFE. Fonte: adaptada de Postma et al. (2012)

\begin{tabular}{|c|c|c|}
\hline Funções do Planejamento de Cenário & $\begin{array}{l}\text { Contri } \\
\text { soluc }\end{array}$ & Problemas do Fuzzy Front End \\
\hline $\begin{array}{l}\text { 1. Avaliação e seleção de estratégias } \\
\text { 2. Integração de vários tipos de } \\
\text { dados orientados para o futuro } \\
\text { 3. Exploração do futuro e } \\
\text { identificação de possibilidades } \\
\text { futuras } \\
\text { 4. Conscientização dos gerentes das } \\
\text { incertezas do ambiente } \\
\text { 5. Ampliação dos modelos mentais } \\
\text { dos gestores } \\
\text { 6. Acionamento e aceleração dos } \\
\text { processos de aprendizado } \\
\text { organizacional. }\end{array}$ & & $\begin{array}{l}\text { Gerenciamento de ideias: } \\
\text { a) A centralidade de ideias } \\
\text { b) O papel e os pontos de vista das } \\
\text { partes interessadas e especialmente } \\
\text { de indivíduos com poder de decisão } \\
\text { c) A incerteza acerca dos resultados } \\
\text { d) A orientação para o problema de } \\
\text { curto prazo } \\
\text { e) A falta de ideias adequadas } \\
\text { Gerenciamento da atenção: } \\
\text { f) A capacidade limitada do } \\
\text { indivíduo } \\
\text { g) A capacidade limitada de grupos } \\
\text { ou de organizações }\end{array}$ \\
\hline
\end{tabular}

\section{METODOLOGIA}

Para a avaliação das contribuições do PC para o FFE, foi realizado um Estudo de Caso (EC) (YIN, 1994) em uma grande multinacional do setor automotivo. A empresa estudada possui mais de 10 mil colaboradores e capacidade produtiva de 800 mil veículos por ano.

O foco do estudo se deu no processo de Revisão do Planejamento Estratégico de Produtos de Longo Prazo (PEP-LP) da empresa, que engloba as atividades diretamente relacionadas ao FFE. A revisão do PEP-LP aconteceu de janeiro a maio de 2016 e considerou o horizonte de planejamento de 2019 a 2025 e o contexto competitivo da América Latina. Ele foi realizado por uma equipe central composta por cinco membros do departamento de Planejamento e Estratégia de Produto da empresa. Uma das autoras desse estudo trabalhava nesse departamento na época da execução do planejamento, o que permitiu que a coleta de dados acontecesse por observação sistemática e não-participante, pesquisa documental e entrevistas em profundidade com os principais participantes do processo. Também foram entrevistadas todas as pessoas que participaram de pelo menos $85 \%$ dos workshops do processo.

Além das entrevistas, os participantes também responderem a um questionário objetivo, realizado ao final do PEP-LP. O questionário era composto por dois grandes blocos, sendo o primeiro relacionado ao processo de aplicação da metodologia GBN e o segundo baseado nos problemas do FFE listados por Postma et al. (2012).

\section{O CASO}

O processo de revisão do PEP-LP na indústria automotiva estudada seguiu as etapas indicadas pela metodologia GBN. O quadro 1 caracteriza cada uma das cinco fases do processo, indicando o número de participantes, os principais inputs e outputs, as ferramentas de apoio utilizadas, além de um comparativo com as atividades previstas pela GBN. Ao todo foram realizados sete workshops de construção de conteúdo, que resultaram em 4 oportunidades de produtos, 4 de novos negócios e 6 de novos serviços.

Antes do início efetivo do PEP-LP, houve uma etapa preliminar para o planejamento e preparação dos workshops e materiais de apoio, e para a seleção dos participantes. O número de participantes, apesar de variar por workshop, foi de 30 pessoas de 15 áreas estratégicas distintas e de diferentes níveis gerenciais, seguindo a indicação de diversidade sugerida pela GBN. 
Quadro 1. Etapas de Execução da Revisão do PEP-LP. Fonte: elaborado pelos autores

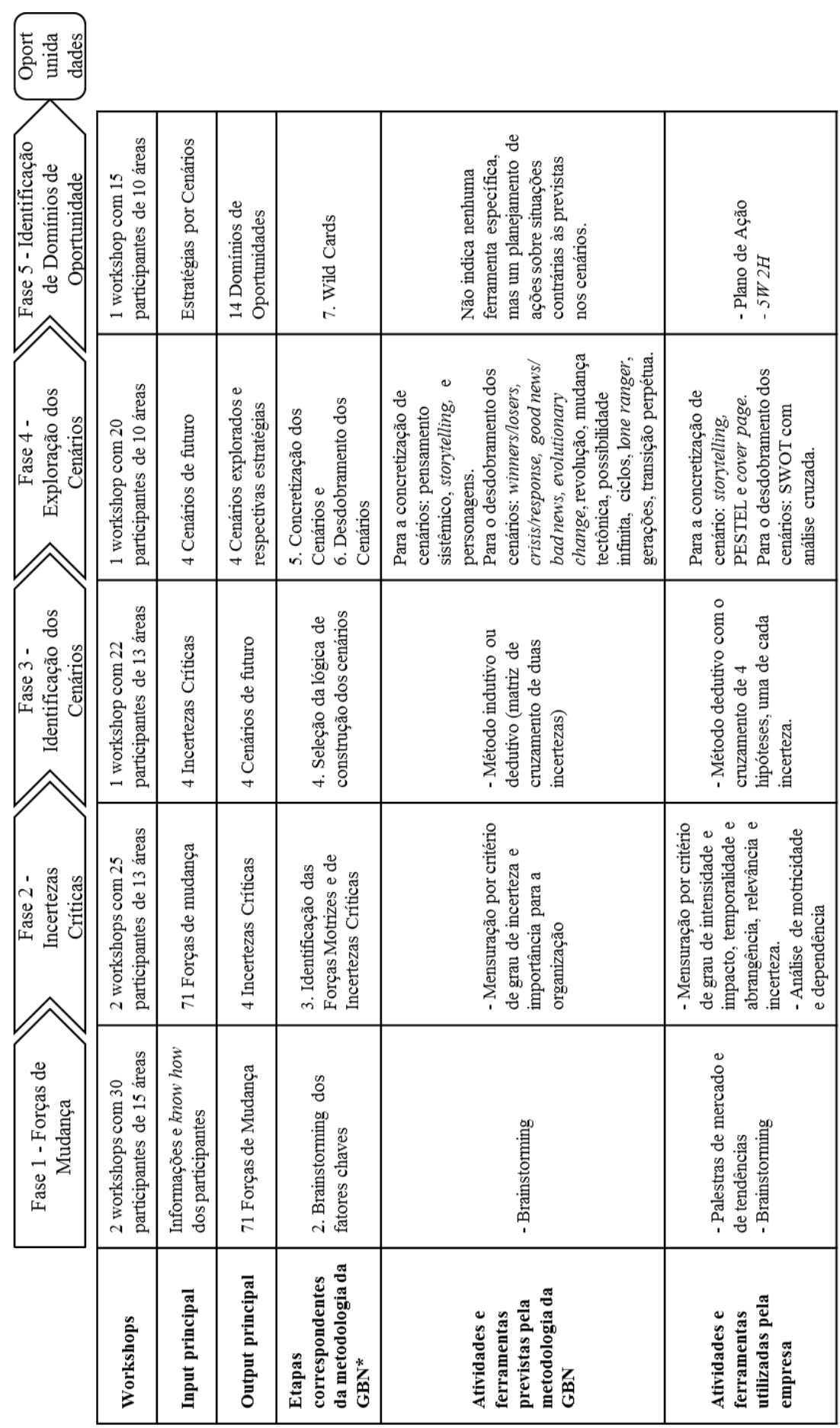

Na fase 1 - Forças de Mudanças, a captura da maior diversidade e quantidade de informações possíveis para sustentar as análises foi um dos grandes desafios. Através da técnica de brainstorming e relatórios e palestras de consultorias externas de mercado, os participantes identificaram 71 Forças de Mudança relevantes, considerando as esferas social, tecnológica, econômica, ambiental e política.

A fase 2 - Incertezas Críticas teve por objetivo identificar dentre as Forças de Mudanças, aquelas que se caracterizam como incertezas críticas para o negócio. A utilização combinada dos critérios de intensidade e impacto, temporalidade e abrangência, relevância e incerteza, e 
motricidade e dependências, permitiu diferenciar as 4 incertezas críticas para a organização, que foram descritas em três possíveis hipóteses de ocorrência.

Na fase seguinte (Fase 3 - Identificação dos Cenários), a combinação aleatória entre todas as hipóteses das quatro incertezas críticas resultou em 81 cenários, cada um deles composto por quatro hipóteses, uma de cada incerteza. A fase incluiu também a avaliação da coerência dos cenários e a sua classificação quanto a probabilidade de ocorrência e impacto para a indústria, permitindo a seleção dos 4 cenários mais relevantes a serem explorados: um cenário de alto impacto para empresa, dois cenários competitivos de média probabilidade e impacto, e um cenário de elevada probabilidade de ocorrência.

Na fase 4 - Exploração de Cenários, a caracterização dos 4 cenários foi realizada através das ferramentas PESTEL, Storytelling e Capa de Revista, utilizadas pelos participantes durante o workshop. Uma vez caracterizados os cenários em narrativas lúdicas e de fácil compreensão, foi realizada uma análise SWOT de cada cenário, o que permitiu a identificação de diferentes estratégias para cada um deles. Assim, foi possível estabelecer prioridades de atuação, fazer diagnósticos e recomendar estratégias.

A Identificação de 14 Domínios de Oportunidade (fase 5) sendo 4 de novos negócios, 4 de novos produtos e 6 de novos serviços, foi realizada a partir da investigação das estratégias para cada um dos cenários e da análise das informações de mercado, das forças de mudança e das necessidades dos clientes. Além does resultados tangíveis do processo (i.e. domínios de oportunidade), os participantes evidenciaram resultados intangíveis, como a visão global do ambiente, a integração e uso da inteligência coletiva, a criação de redes de informação e a mudança de mapas mentais. Seis meses após o fim do processo, duas das quatro oportunidades de produtos já estavam em estudo para compor efetivamente o PEP-LP da empresa, e as demais já estavam em etapa de avaliação.

\section{DISCUSSÕES}

O gráfico 1 apresenta a avaliação dos participantes dos workshops em relação aos problemas do FFE listados por Postma et al. (2012). Observa-se que 100\% dos entrevistados concordam totalmente que o número de ideias discutidas foi influenciado positivamente pelo PC da GBN. Além disso, $83 \%$ dos participantes concordam totalmente que a utilização da metodologia teve impacto positivo no que tange a descentralização da geração de ideias e captura de oportunidades, o aumento do foco em problemas a longo prazo e o aumento da robustez dos resultados devido ao processo ter sido multifuncional e colaborativo. Por outro lado, a suavização na maneira top-down de tomada de decisões estratégicas e a redução do impacto de incertezas futuras não foram um consenso entre os respondentes.

Dessa forma, conclui-se que a utilização do PC contribuiu para reduzir os problemas apresentados por Postma et al. (2012) (ver figura 2): a) centralidade de ideias, d) orientação para o problema de curto prazo, f) capacidade limitada do indivíduo; e) falta de ideias adequadas, e g) capacidade limitada de grupos ou de organizações, mas não os problemas: b) o papel e os pontos de vista das partes interessadas e especialmente de indivíduos com poder de decisão, e c) a incerteza acerca dos resultados, corroborando parcialmente com os resultados encontrados pelos autores. 
Gráfico 1. Resultados do impacto do PC nos problemas do FFE. Fonte: elaborado pelos autores

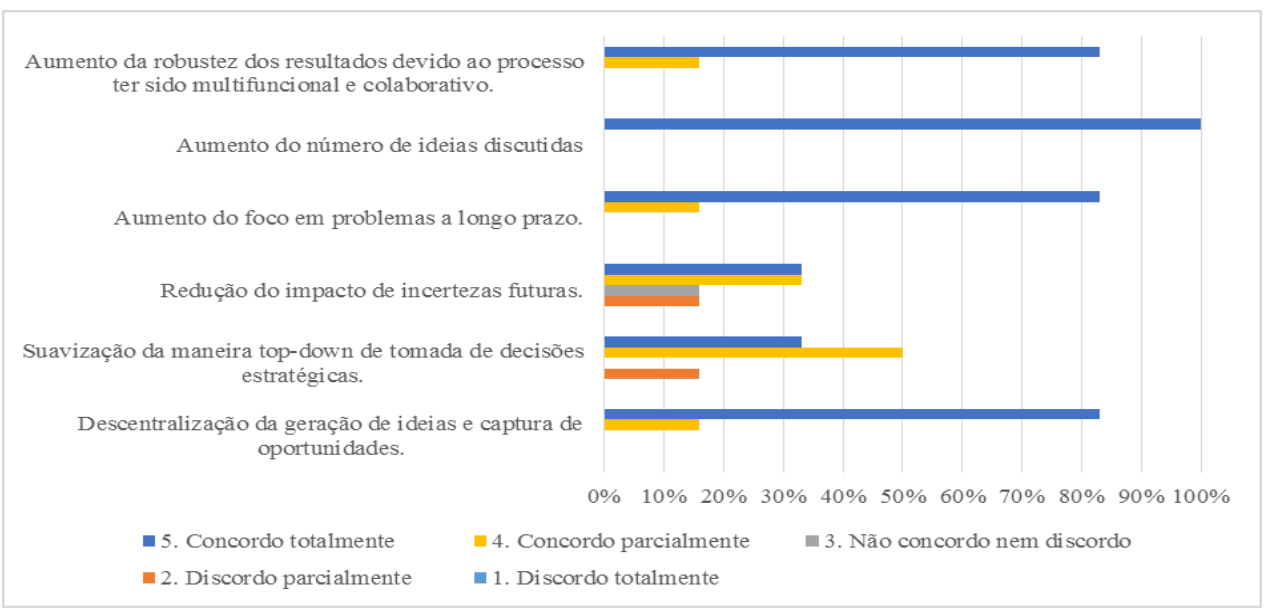

Em relação à análise do processo de aplicação da metodologia da GBN, alguns pontos críticos puderam ser identificados e são citados no Quadro 2. Destaca-se, entre outros: a necessidade de definição de gatilhos que indiquem a ocorrência de cada cenário e formas de monitorá-los; e a contribuição que palestras de consultorias externas tiveram na fase inicial, como forma de provocar os participantes e eliminar gaps de conhecimento. Considerando os pontos de melhoria identificados pelos participantes, destaca-se à sugestão de um intervalo de tempo menor para execução do processo, o que pode implicar na redução do número de workshops e na maior assiduidade dos participantes.

Quadro 2. Pontos críticos para a aplicação da GBN no PEP-LP. Fonte: elaborado pelos autores

\begin{tabular}{|c|c|c|c|c|}
\hline Pesquisa e Preparação & \multicolumn{3}{|c|}{ Execução da Metodologia } & \multirow{3}{*}{$\begin{array}{l}\text { Integração do estudo de } \\
\text { cenários ao } \\
\text { planejamento estratégico }\end{array}$} \\
\hline & Imersão & Análise & Decisão & \\
\hline $\begin{array}{l}\text { Etapa do Estudo de Caso: } \\
\text { 4.2 Planejamento do processo de } \\
\text { revisão do LRP utilizando a } \\
\text { GBN }\end{array}$ & $\begin{array}{l}\text { Etapa do Estudo de Caso: } \\
\text { 4.3.1 Forças de Mudança } \\
\text { 4.3.2 Incertezas Críticas } \\
\text { 4.3.3 Identificação de Cenários }\end{array}$ & $\begin{array}{l}\text { Etapa do Estudo de Caso: } \\
\text { 4.3.4 Exploração de Cenários }\end{array}$ & $\begin{array}{l}\text { Etapa do Estudo de Caso: } \\
\text { 4.3.5 Identificação de Domínios } \\
\text { de Oportunidades }\end{array}$ & \\
\hline $\begin{array}{l}\text { Pontos Críticos: } \\
\text { i. Relevância da permanência de } \\
\text { um core team durante todo o } \\
\text { projeto } \\
\text { ii. Necessidade da presença de } \\
\text { uma equipe multifuncional e de } \\
\text { diferentes niveis organizacionais } \\
\text { iii. Importância do planejamento } \\
\text { detalhado do processo para evitar } \\
\text { atrasose e ausências. } \\
\text { iv. Internalização da metodologia } \\
\text { como forma de redução de custo } \\
\text { e de obtenção de conhecimento } \\
\text { v. Atenção para que seja } \\
\text { realizado o processo uma época } \\
\text { do ano onde exista menor } \\
\text { concorrência com outros projetos } \\
\text { vi. Importância de materiais } \\
\text { prévios aos WS para } \\
\text { nivelamento de conhecimentos }\end{array}$ & $\begin{array}{l}\text { Pontos Críticos: } \\
\text { vii. Importância de palestras para } \\
\text { instigar e provocar os } \\
\text { participantes } \\
\text { viii. Identificação de } 4 \text { como o } \\
\text { número de cenários indicados } \\
\text { para exploração }\end{array}$ & $\begin{array}{l}\text { Pontos Críticos: } \\
\text { ix. Necessidade de investigar } \\
\text { triggers que indicam a } \\
\text { ocorrência de cada cenário } \\
\text { x. Relevância de um } \\
\text { monitoramento acessivel dos } \\
\text { triggers identificados } \\
\text { xi. Importância da criação de } \\
\text { planos de ação no caso de } \\
\text { ativação de um dos cenários }\end{array}$ & $\begin{array}{l}\text { Pontos Críticos: } \\
\text { xii. Priorização a diversidade de } \\
\text { áreas envolvidas e a presença de } \\
\text { representantes seniores para } \\
\text { enriquecimento da identificação } \\
\text { de oportunidades } \\
\text { xiii. Atenção para que os } \\
\text { resultados do processo sejam } \\
\text { apresentados às áreas envolvidas }\end{array}$ & $\begin{array}{l}\text { Pontos Críticos: } \\
\text { xiv. Destaque do } \\
\text { comprometimento sênior com a } \\
\text { continuidade da metodologia e } \\
\text { desdobramento de oportunidades } \\
\text { xv. Relevância de se considerar } \\
\text { as implicações de cenários ao } \\
\text { tomar decisões estratégicas e/ou } \\
\text { a longo prazo } \\
\text { xvi. Importância da divulgação } \\
\text { do processo para outras áreas }\end{array}$ \\
\hline
\end{tabular}

Para os participantes "a oportunidade de compartilhar conhecimentos de diferentes áreas $e$ pontos de vistas ajudou a construir uma visão sobre um futuro em que todos compartilham" (designer automotivo) e "ampliar o olhar para o que está acontecendo, conversar com outras pessoas fazerem as famosas conexões improváveis" (entrevistado 2 - coordenadora de gestão da inovação). Além disso, $83 \%$ dos entrevistados adotariam a metodologia para a próxima revisão do LRP, um indicativo de que o PC poderia ser implementado de maneira definitiva na empresa, "porque é uma metodologia consolidada, apresenta resultados consistentes, 
provoca oxigenação nas pessoas e reduz o automatismo do dia-a-dia ao deslocar os participantes do seu ambiente de trabalho para pensar em outro propósito" (coordenadora de gestão da inovação).

Considerando que anteriormente o planejamento estratégico de produtos da empresa era realizado por poucos indivíduos e restrito a apenas duas áreas, a metodologia de PC ampliou consideravelmente o escopo e os modelos mentais, de modo a tornar o processo muito mais colaborativo.

\section{CONCLUSÕES}

Diante do elevado nível de incerteza e complexidade associado ao FFE e da sua importância para o sucesso de novos produtos, vários processos, técnicas e ferramentas são propostos para auxiliar no gerenciamento e sucesso da fase. A partir de um estudo de caso em uma grande indústria automotiva, este artigo apresenta em detalhe como uma metodologia específica de PC foi utilizada para guiar as atividades associadas ao FFE e avalia como sua aplicação reduziu alguns dos problemas associados à fase a partir da percepção dos colaboradores da empresa.

Em comparação ao trabalho de Postma et al. (2012), os resultados encontrados reforçam que o PC contribui positivamente para a redução da centralidade de ideias e da orientação para problemas de curto prazo, e para o aumento do número e da robustez das ideias criadas. Entretanto, não foram obtidas evidências conclusivas de que o método reduz o impacto das incertezas futuras e suaviza a maneira top-down de tomada de decisões estratégicas, conforme previsto pela literatura. De forma geral, a metodologia permitiu a criação de um processo colaborativo, no qual a participação de pessoas de diferentes áreas foi essencial.

Do ponto de vista prático, os resultados apresentados podem orientar os gestores a iniciarem a utilização do PC nos seus processos de desenvolvimento de produtos. Além de descrever o processo, foram identificados diversos pontos de melhoria e atenção, como a necessidade de identificação e monitoramento de gatilhos que indiquem a ocorrência dos cenários previstos e a necessidade do maior envolvimento de participantes sêniores durante todo o processo.

Apesar do setor automotivo ser proeminente quanto adoção de métodos de PDP e apresentar um contexto de crescente importância da fase de pré-desenvolvimento, este trabalho limitouse a um estudo de caso único. Sugere-se que estudos futuros sejam realizados em outros casos com diferentes contextos para validar os resultados encontrados.

\section{REFERÊNCIAS}

BACKMAN, M.; BÖRJESSON, S.; SETTERBERG, S. Working with concepts in the fuzzy front end: exploring the context for innovation for different types of concepts at Volvo Cars. R\&d Management, v. 37, n. 1, p. 17-28, 2007. ISSN 1467-9310.

BISHOP, P.; HINES, A.; COLLINS, T. The current state of scenario development: an overview of techniques. foresight, v. 9, n. 1, p. 5-25, 2007. ISSN 1463-6689.

BOOD, R.; POSTMA, T. Strategic learning with scenarios. European Management Journal, v. 15, n. 6, p. 633-647, 1997. ISSN 0263-2373.

COOPER, R.G. Predevelopment activities determine new product success. Industrial Marketing Management, v. 17, n. 3, p. 237-247, 1988. ISSN 0019-8501. 
COSTA, M.A.B.; TOLEDO, J.C.D. Analisis of predevelopment models and activities: a systematic bibliografic review. Gestão \& Produção, n. AHEAD, p. 0-0, 2016. ISSN 0104$530 \mathrm{X}$.

HOLAHAN, P.J.; SULLIVAN, Z.Z.; MARKHAM, S.K. Product development as core competence: how formal product development practices differ for radical, more innovative, and incremental product innovations. Journal of Product Innovation Management, v. 31, n. 2, p. 329-345, 2014. ISSN 1540-5885.

JETTER, A.J.M. Educating the guess: strategies, concepts and tools for the fuzzy front end of product development. Management of Engineering and Technology, 2003. PICMET'03. Technology Management for Reshaping the World. Portland International Conference on, 2003. IEEE. p.261-273.

KATZ, G. Rethinking the product development funnel. Visions, v. 35, n. 2, p. 24-31, 2011.

KHURANA, A.; ROSENTHAL, S.R. Towards holistic "front ends" in new product development. Journal of Product Innovation Management, v. 15, n. 1, p. 57-74, 1998. ISSN 1540-5885.

KIM, J.; WILEMON, D. Focusing the fuzzy front-end in new product development. R\&D Management, v. 32, p. 269-279, 2002.

KOEN, P. et al. Providing clarity and a common language to the" fuzzy front end". Research Technology Management, v. 44, n. 2, p. 46, 2001. ISSN 0895-6308.

MIGUEL, P.A.C. The potential of new product development in the automotive industry in Brazil: an exploratory study. Product: Management \& Development, v. 4, n. 1, p. 35-43, 2006.

MOENAERT, R.K. et al. R\&D/marketing communication during the fuzzy front-end. Engineering Management, IEEE Transactions on, v. 42, n. 3, p. 243-258, 1995. ISSN 00189391.

OGILVY, J.; SCHWARTZ, P. Plotting your scenarios 2004.

POSTMA, T.J.B.M.; BROEKHUIZEN, T.L.J.; VAN DEN BOSCH, F. The contribution of scenario analysis to the front-end of new product development. Futures, v. 44, n. 6, p. 642654, 2012. ISSN 0016-3287.

SCHOEMAKER, P.J.H. Scenario planning: a tool for strategic thinking. Sloan management review, v. 36, n. 2, p. 25, 1995. ISSN 0019-848X.

STURGEON, T.; VAN BIESEBROECK, J.; GEREFFI, G. Value chains, networks and clusters: reframing the global automotive industry. Journal of economic geography, v. 8, n. 3, p. 297-321, 2008. ISSN 1468-2702.

TIDD, J.; BESSANT, J.; PAVITT, K. Gestão da Inovação. Porto Alegre: Bookman, 2008. 600p. ISBN 9788577802029.

VAN DE VEN, A.H. Central problems in the management of innovation. Management science, v. 32, n. 5, p. 590-607, 1986. ISSN 0025-1909.

VAN DER HEIJDEN, K. Scenarios: the art of strategic conversation. 1996.

YIN, R.K. Case study research: design and methods. Sage Publications, 1994.

WACK, P. Uncharted waters ahead. Harvard Business Review, 5, September/October, 1985. 